The geometrical nature of optical resonances: from a sphere to fused dimer nanoparticles

This article has been downloaded from IOPscience. Please scroll down to see the full text article.

2012 Meas. Sci. Technol. 23084002

(http://iopscience.iop.org/0957-0233/23/8/084002)

View the table of contents for this issue, or go to the journal homepage for more

Download details:

IP Address: 130.159.216.8

The article was downloaded on 22/11/2012 at 15:01

Please note that terms and conditions apply. 


\title{
The geometrical nature of optical resonances: from a sphere to fused dimer nanoparticles
}

\author{
B Hourahine and F Papoff \\ SUPA, Department of Physics, University of Strathclyde, 107 Rottenrow, Glasgow G4 0NG, UK \\ E-mail: benjamin.hourahine@strath.ac.uk and papoff@phys.strath.ac.uk
}

Received 1 December 2011, in final form 1 February 2012

Published 19 June 2012

Online at stacks.iop.org/MST/23/084002

\begin{abstract}
We study the electromagnetic response of smooth gold nanoparticles with shapes varying from a single sphere to two ellipsoids joined smoothly at their vertices. We show that the plasmonic resonance visible in the extinction and absorption cross sections shifts to longer wavelengths and eventually disappears as the mid-plane waist of the composite particle becomes narrower. This process corresponds to an increase of the numbers of internal and scattering modes that are mainly confined to the surface and coupled to the incident field. These modes strongly affect the near field, and therefore are of great importance in surface spectroscopy, but are almost undetectable in the far field.
\end{abstract}

Keywords: light scattering, absorption, gold nanoparticles, theory

(Some figures may appear in colour only in the online journal)

\section{Introduction}

The interaction of light with micro- and nano-particles is the basis of many important effects and applications, such as single molecule spectroscopy and photon processes [1-3]. Recently, we have developed a theory that gives a geometrical interpretation of the resonances of the Maxwell equations by using eigenvalues and eigenvectors of Hermitian operators which are similar in form to those used in quantum mechanics [4]. In this paper, we apply this theory to study the effect of shape on the electromagnetic response of gold particles varying from a sphere to two ellipsoids joined together at their vertices and forming a fused dimer. Fused dimers have been extensively investigated in view of application to surfaceenhanced spectroscopy, using a model of two intersecting spheres, with the distance between the two centres less than the sum of the radii $[5,6]$. For light incidence perpendicular to the axis of the dimer with its electric field parallel to the distance between the centres, resonances are blue-shifted as the distance between the centres and the length of the dimer decreases. The opposite effect happens for polarization perpendicular to the axis as the length of the structure grows. Instead, in this paper, we keep the length of the dimer constant and analyse the variation of near and far-field optical properties as a function of the depth of the indentation.

\section{Theory}

We consider metallic particles without sharp edges and of dimensions of the order of the wavelength of the incident field or smaller, so that the interaction between light and matter inside the particle is described by the local macroscopic permittivity and susceptibility. The tangential components of electric and magnetic fields $(E, H)$ are continuous on passing through particle boundaries, and the energy scattered by a particle flows towards infinity. We use six-component vectors, $F=[E, H]^{T}$, for electromagnetic fields [7]; their projections, $f$, onto the surface of the particle are surface fields that form a space $\mathcal{H}$ where scalar products are defined in terms of integrals over the particle surface of the form $f \cdot g=\sum_{j} \int_{S} f_{j}^{*} g_{j} \mathrm{~d} s$, where the index $j$ labels the components of the fields and $f^{*}$ is the complex conjugate of $f$. In this formalism, the electromagnetic boundary conditions are reduced to the single equation

$$
f^{0}=f^{i}-f^{s}
$$




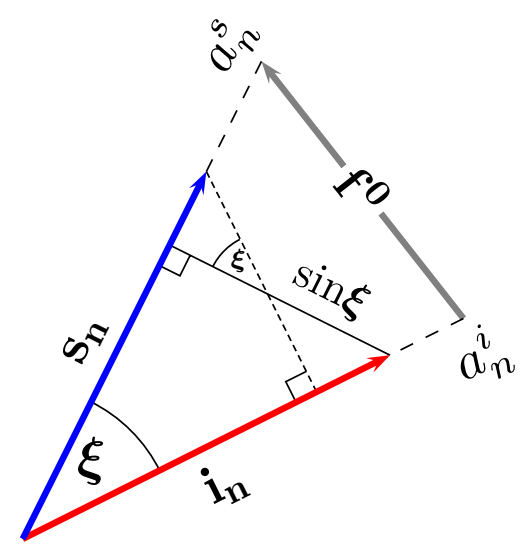

Figure 1. An internal $\left(i_{n}\right)$ and scattered $\left(s_{n}\right)$ principal mode pair and their coupling to part of the incident light, leading to their amplitudes for this particular incident field $\left(a_{n}^{i}\right.$ and $\left.a_{n}^{s}\right)$. The angle between the modes, $\xi$, controls both the amplitude of the modes and the correlation between them.

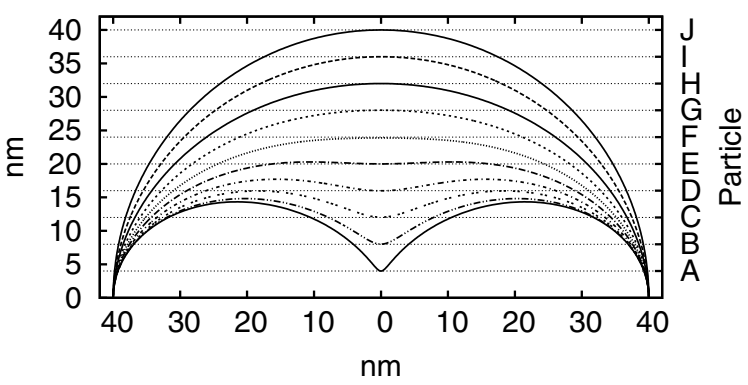

Figure 2. Cross-sectional geometries of the ten particles considered in this work, labelled A-J, depending on the depth of the indentation, ranging from touching spheroids (A) through a rounded cylinder $(\mathrm{F})$ to a sphere $(\mathrm{J})$.

which has a simple geometrical meaning in $\mathcal{H}$ : the projection, $f^{0}$, of the incident field onto the surface is equal to the difference between the projections of the internal and scattered fields, $f^{i}$ and $f^{s}$. As a consequence, an external field with small tangential components can excite large internal and scattered surface fields provided that these closely cancel each other. For particles without sharp edges, we can form separate orthonormal bases $\left\{i_{n}\right\}$ and $\left\{s_{n}\right\}$ for the projections of internal and scattered fields. These fields, which we call principal internal and scattering modes, are orthogonal to all but at most one function in the other space (see figure 1). For spherical particles, each pair of modes corresponds to a pair of electric or magnetic multipoles of Mie theory. For non-spherical particles, the principal modes are instead combinations of electric and magnetic multipoles (although in some cases there can be dominant contributions from a specific multipole). For all axially symmetric particles, including the cases considered in this paper, modes have an $\exp i l_{z} \phi$ dependence on the longitudinal angle $\phi$ around the symmetry axis, where $l_{z}$ can be any integer number. This allows the resolution of modes into groups having different angular momenta, $l_{z}$.

We can find the internal and scattered fields for a particular incident field by decomposing it into a sum of pairs of principal modes, $f^{0}=\sum_{n} a_{n}^{i} i_{n}-a_{n}^{s} s_{n}$, where $a^{i / s}$ are amplitudes of each of the internal and scattered principal modes for

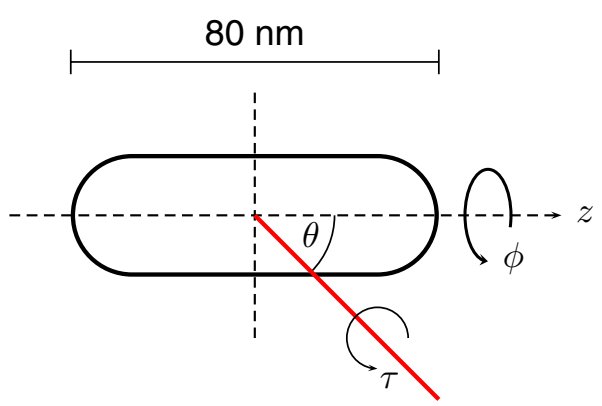

Figure 3. Schematic of the scattering geometry, with the $\theta$ and $\phi$ angles marked. Incident plane waves are oriented with an angle of $\theta$ with respect to the $z$ axis (at $\theta=0$ ) and an electric field polarization $\tau$ with respect to the $z$ axis (again at $\tau=0^{\circ}$ ).
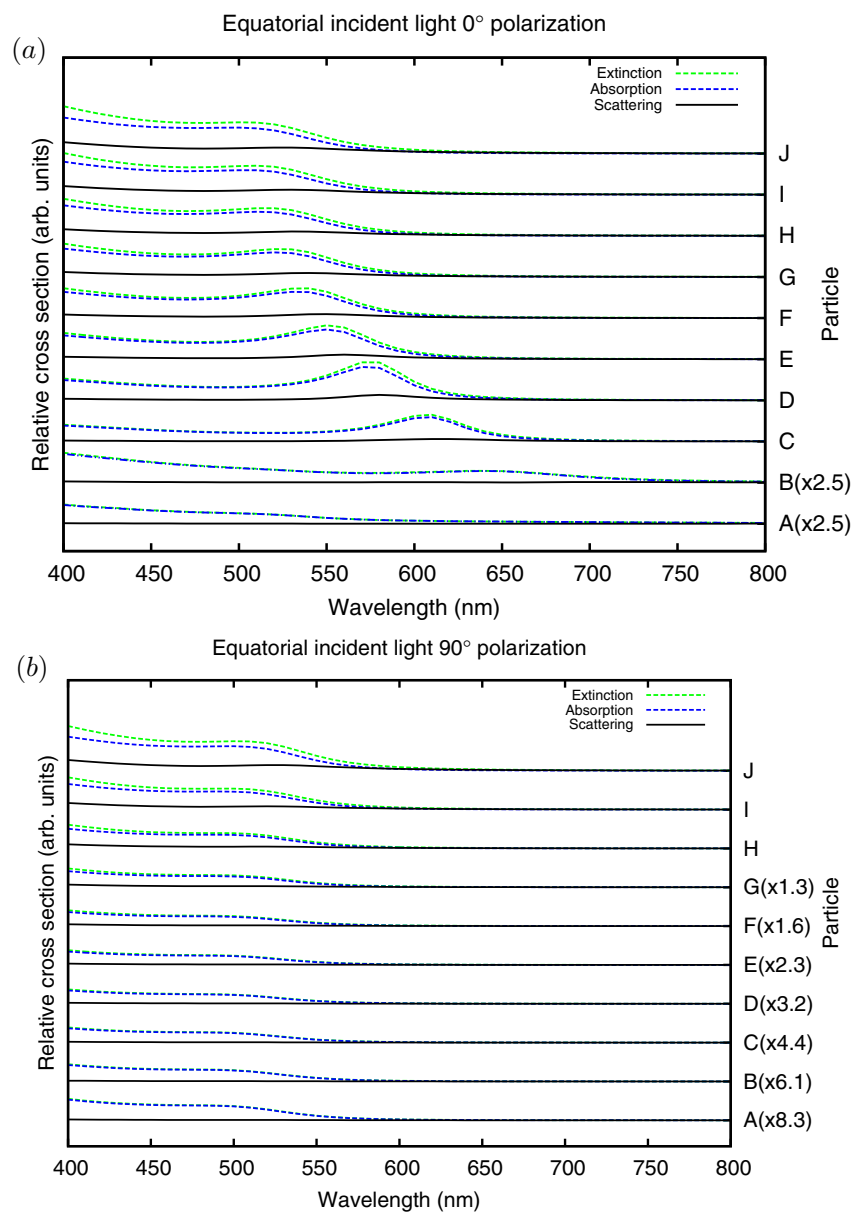

Figure 4. Optical cross sections for particles A-J for $\theta=90^{\circ}$ illumination with the electric field component $(a)$ parallel to the particle axis and $(b)$ perpendicular.

that specific incident field. The statistical correlation [8] and the orthogonal distance between $s_{n}$ and $i_{n}$ in $\mathcal{H}$, which are calculated through the overlap integral of the modes on the surface of the particle [4], are the so-called principal cosines and sines, $\cos \left(\xi_{n}\right)$ and $\sin \left(\xi_{n}\right)$. The angles $\xi$ are invariant under unitary transformation and characterize the geometry of the subspaces of the internal and scattered solutions in $\mathcal{H}$. This geometry is induced by the particular particle through the surface integrals [4]. Principal cosines provide analytic equations for the coefficients of the internal and scattered 


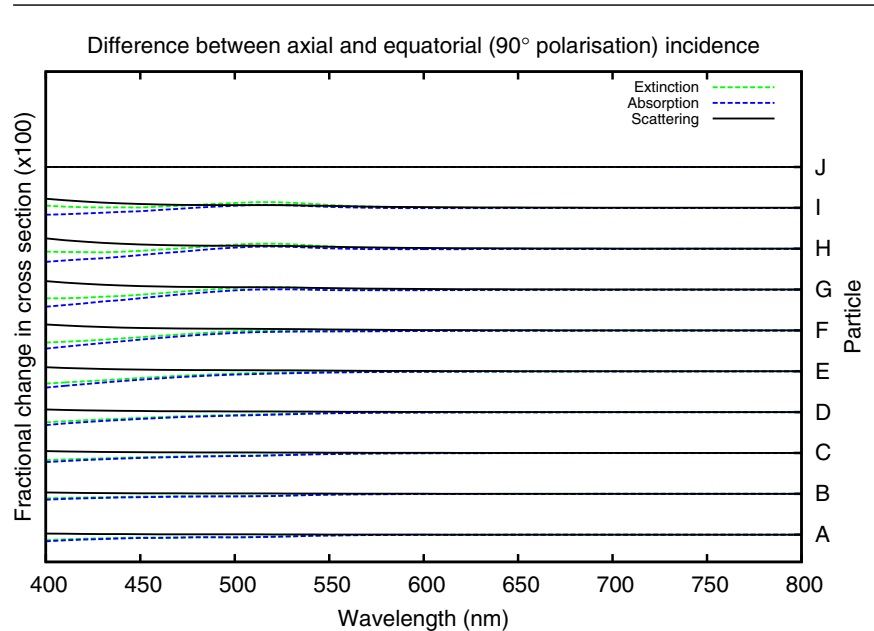

Figure 5. Difference in optical cross sections between $\theta=0^{\circ}$ incident light (of any polarization) and $\theta=90^{\circ}, \tau=90^{\circ}$ illumination (with that cross section shown in figure $4(b)$ ). The maximum difference in the extinction cross section is $\approx 4 \%$ between the two types of incident light.

principal modes, generalizing the Mie formulae and clarifying the nature of all scattering channels, and eliminate the need for numerical inversion for the determination of the mode coefficients. The coefficients $a_{n}^{i}, a_{n}^{s}$ of the principal modes are found by projecting the incident field $f^{0}$ onto non-orthogonal vectors $i_{n}, s_{n}$ and are

$$
\begin{gathered}
a_{n}^{i}=\frac{i_{n}-\cos \left(\xi_{n}\right) s_{n}}{\sin ^{2}\left(\xi_{n}\right)} \cdot f^{0}=\frac{i_{n}^{\prime}}{i_{n}^{\prime} \cdot i_{n}} \cdot f^{0}, \\
a_{n}^{s}=-\frac{s_{n}-\cos \left(\xi_{n}\right) i_{n}}{\sin ^{2}\left(\xi_{n}\right)} \cdot f^{0}=-\frac{s_{n}^{\prime}}{s_{n}^{\prime} \cdot s_{n}} \cdot f^{0} .
\end{gathered}
$$

Here, $i_{n}^{\prime}=i_{n}-\cos \left(\xi_{n}\right) s_{n}, s_{n}^{\prime}=s_{n}-\cos \left(\xi_{n}\right) i_{n}$ with $i_{n}^{\prime} \cdot s_{n}=$ $s_{n}^{\prime} \cdot i_{n}=0$ and $i_{n}^{\prime} \cdot i_{n}=s_{n}^{\prime} \cdot s_{n}=\sin ^{2}\left(\xi_{n}\right) \cdot \sin \left(\xi_{n}\right)$ is defined as the Peterman factor [9] that gives the order of magnitude of transient gain and excess noise in unstable cavity modes. Therefore, large surface fields in nanoparticles and large transient gain and excess noise in macroscopic unstable cavities and dissipative systems [10-13] have the same geometrical origin.

The surface modes and the amplitudes excited by an incident field fully specify the electromagnetic behaviour of the particle everywhere in space. Moreover, the left-hand terms in the scalar products of equations (2) and (3) depend exclusively on the particle, and this allows us to disentangle its intrinsic properties from those due to the specific incident field. The principal modes and their scalar products depend on the frequency-dependent permittivity and susceptibility of
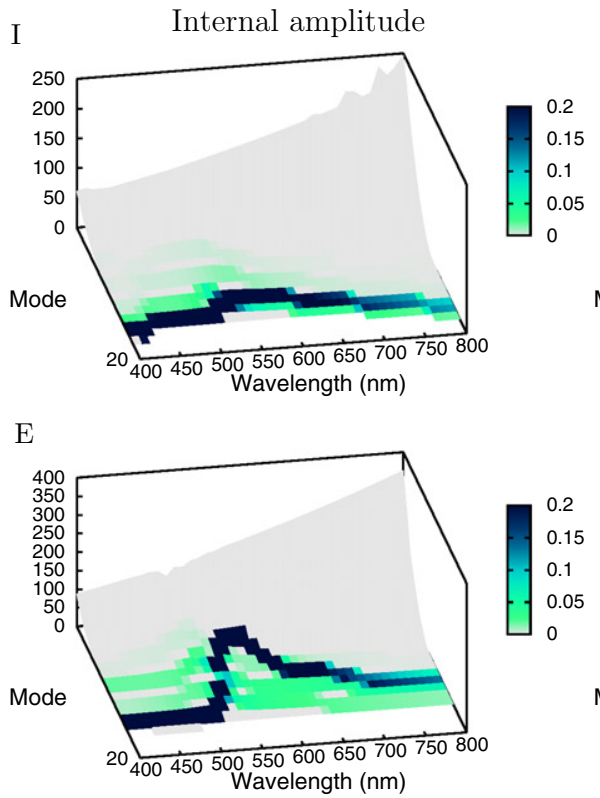

A
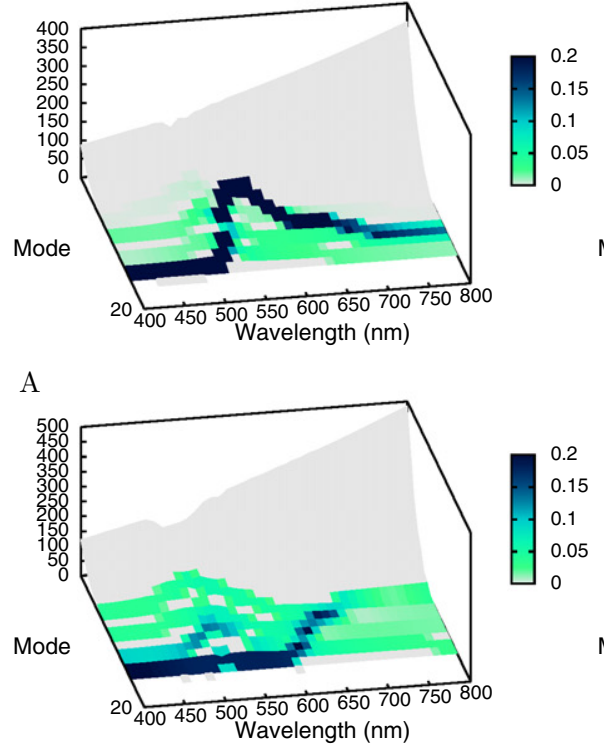

Flux carrying ability
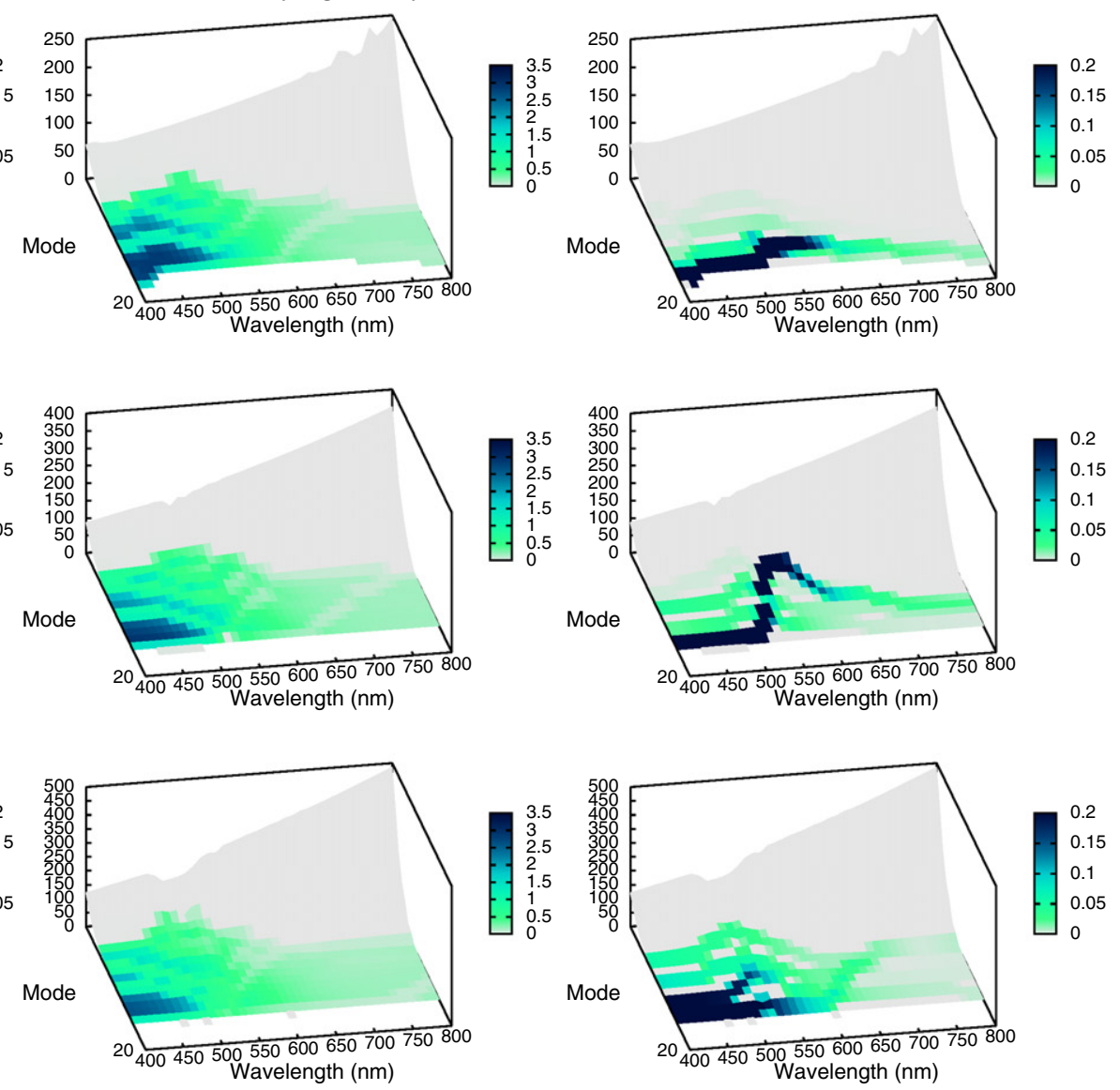
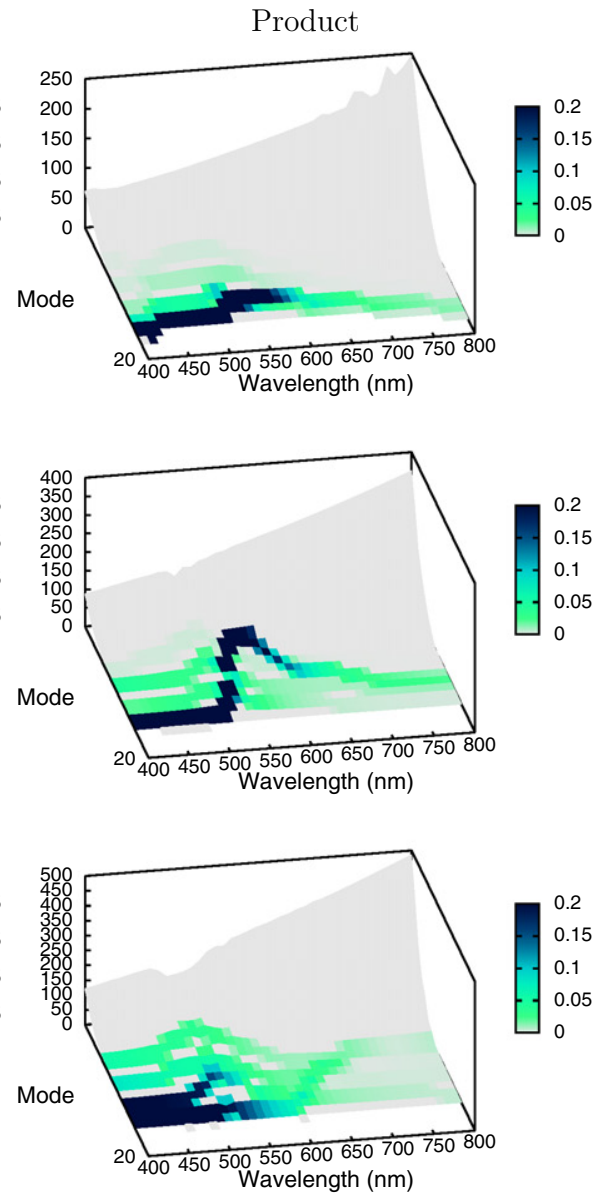

Figure 6. The $l_{z}=0$ internal mode landscapes of selected particles (A, E and I). These are overlaid with traces showing the amplitudes of the modes excited by light incident with $\theta=0^{\circ}$ and polarized with $\tau=90^{\circ}$, their intrinsic ability to transport energy into the particle, and the product of these two (the resulting effective flux being absorbed by each mode). 

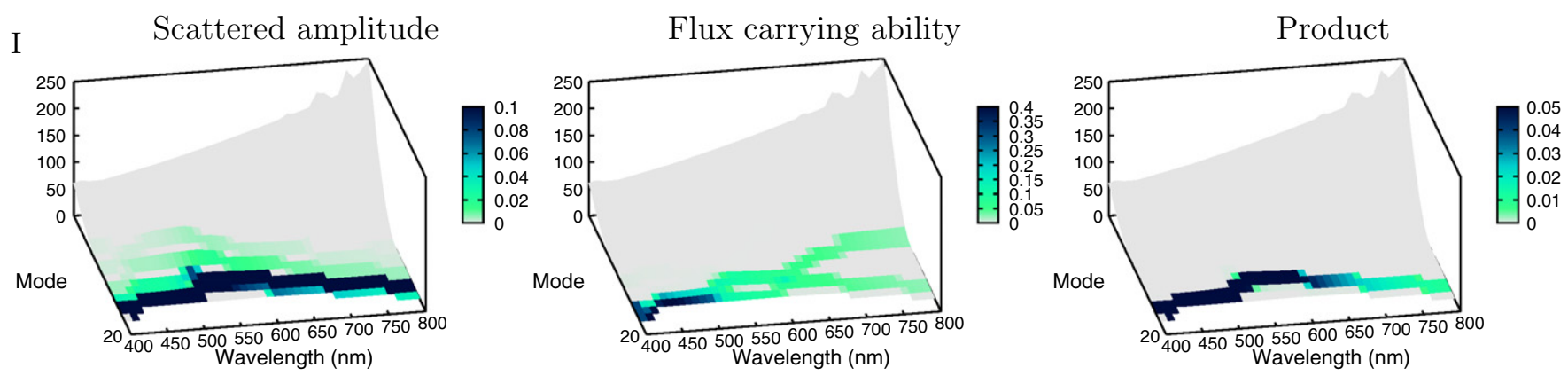

$\mathrm{E}$
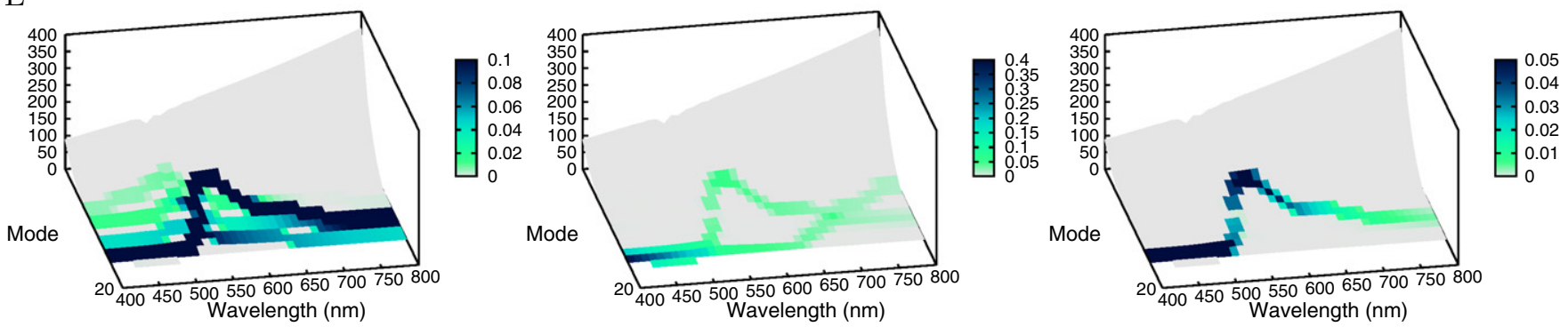

A
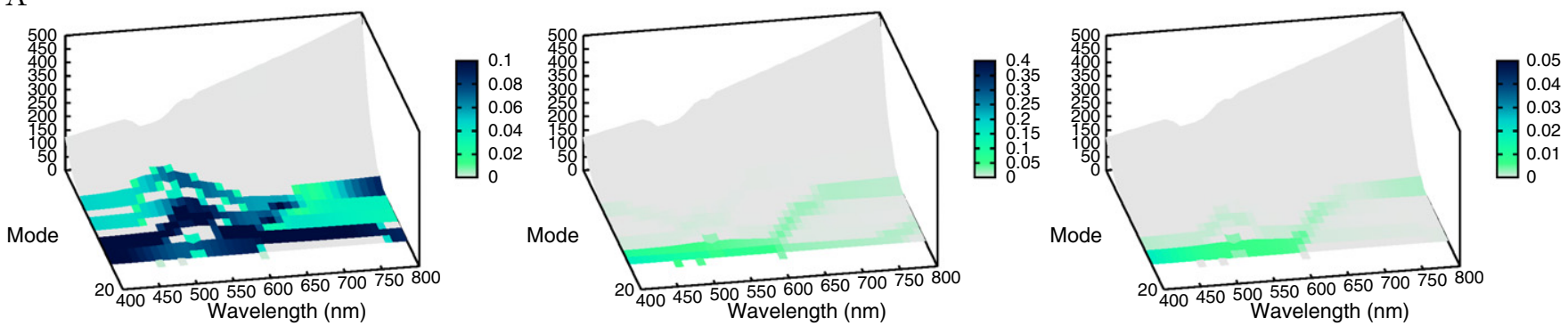

Figure 7. The $l_{z}=0$ scattering mode landscapes of the particles. These are again overlaid with traces showing the amplitudes of the modes excited by light with $\theta=0^{\circ}$ incidence and electric field along the particle axis, their intrinsic ability to transport energy into the particle and the resulting effective flux being scattered away by each mode.

the particle; therefore, the principal angles $\left\{\xi_{n}\right\}$ change with the frequency of incident light. Resonances correspond to minimum angles $\left(\xi_{n} \neq 0\right)$ of pairs in $\mathcal{H}$, and this generalizes the Mie resonance condition to any smooth non-spherical particle [4]. For well-aligned modes, the coefficient $a^{i}, a^{s}$ are of the same order, but this is not the case for weak resonances, which can have qualitatively different absorption and differential scattering cross sections (DSCS). Another important property of principal modes is their ability to transport energy (flux carrying ability), which is given for each mode by its Poynting vector integrated over the surface of the particles, i.e. the energy flux for $\left|a^{i}\right|=\left|a^{s}\right|=1$. For a non-spherical particle, it is possible to have fields which show large components tangent to the particles' surface but that transport very little energy. This means that for such a particle, there can be significant features of the near field that do not appear in the far field.

\section{Results}

We analyse gold particles with a long axis of $80 \mathrm{~nm}$ and shapes ranging from a sphere to a composite particle made
Table 1. Supershape parameters for equation (4) to produce the particles shown in figure 2. All shapes apart from the sphere, $J$, are described by the same superformula with parameters $m=2$, $a=b=1$ and $10 n_{1}=n_{2}=n_{3}$ [14]. $\mathrm{J}$ can be represented as $m=2$, $a=b=1$ and $n_{1}=n_{2}=n_{3}=2$.

\begin{tabular}{llllll}
\hline & $n_{1}$ & & $n_{1}$ & & $n_{1}$ \\
\hline $\mathrm{A}$ & 0.596 & $\mathrm{D}$ & 0.272 & $\mathrm{G}$ & 0.2229 \\
$\mathrm{~B}$ & 0.373 & $\mathrm{E}$ & 0.250 & $\mathrm{H}$ & 0.2138 \\
$\mathrm{C}$ & 0.306 & $\mathrm{~F}$ & 0.235 & $\mathrm{I}$ & 0.2063 \\
\hline
\end{tabular}

by two spheroids merged near to their vertices. A single 'superformula' [14]

$$
r(\theta)=\left(\left|\frac{\cos (m \theta / 4)}{a}\right|^{n_{2}}+\left|\frac{\sin (m \theta / 4)}{b}\right|^{n_{3}}\right)^{-1 / n_{1}}
$$

is used to describe all the generatrices shown in figure 2, with the specific particle parameters given in table 1. Each particle is smooth (a $C_{2}$ continuous surface) and the error in fulfilling the boundary condition of equation (1) therefore gives an upper bound on the difference between the exact and calculated values for the internal and scattered fields everywhere in space [15]. The dielectric function for gold is taken from the literature $[16,17]$. We excite the particles with plane wave light with wavelengths between 400 and $800 \mathrm{~nm}$, 

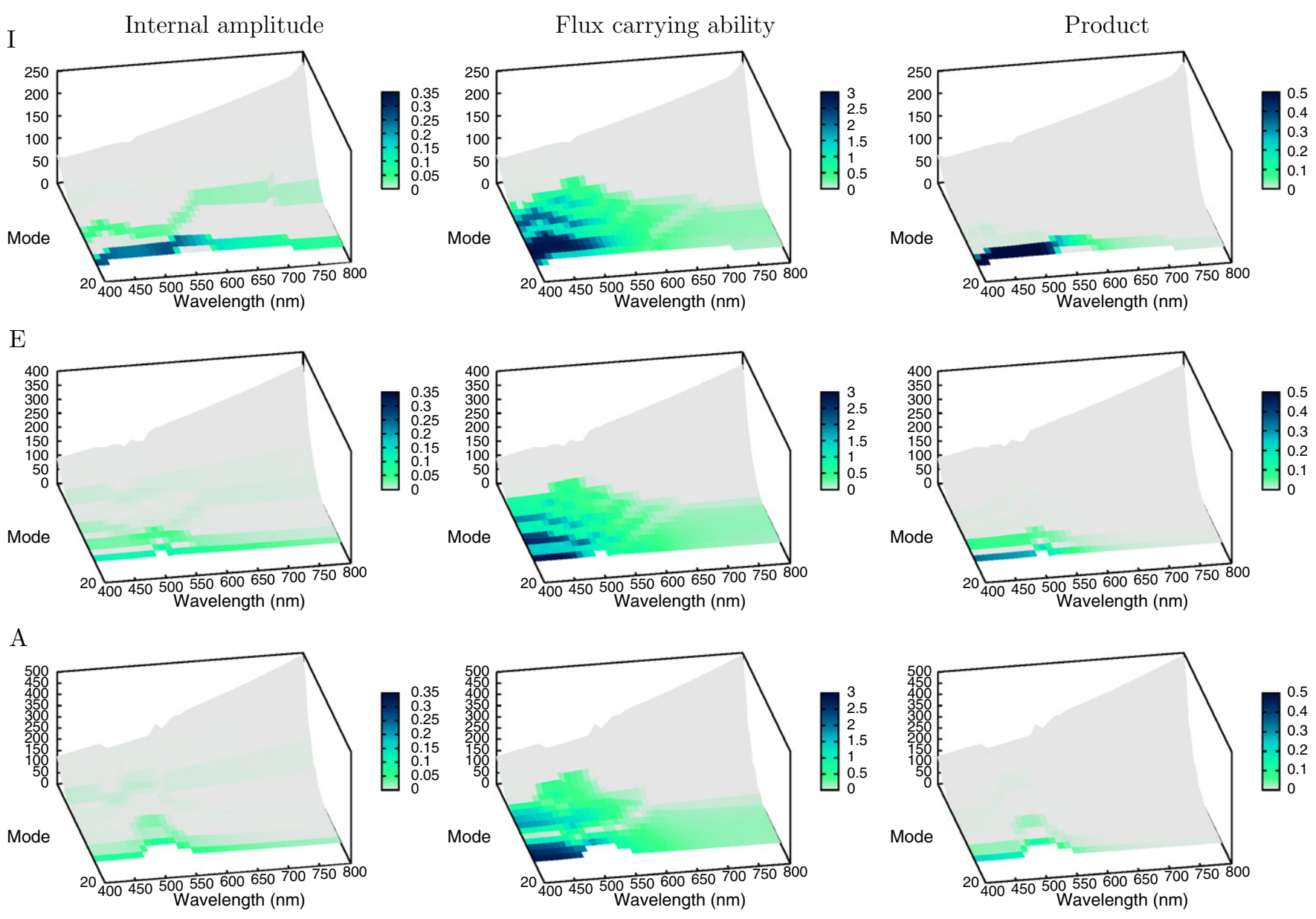

Figure 8. The $l_{z}= \pm 1$ internal mode landscapes of selected particles (A, E and I). These are overlaid with traces showing the amplitudes of the modes excited by light incident equatorially $\left(\theta=90^{\circ}\right)$ and polarized with the electric field perpendicular to the particle axis $\left(\tau=90^{\circ}\right)$, their intrinsic ability to transport energy into the particle and the resulting effective flux being absorbed by each mode.

with the scattering geometry shown schematically in figure 3. Plane waves contain components with all values of $l_{z}$, but for axially symmetric particles with light incident along $\theta=0$, only the $l_{z}= \pm 1$ part of the light can couple with the particle [15].

Normalized extinction, absorption and scattering cross sections are given in figure 4 for incident light aligned normal to the axis of symmetry of the particles $\left(\theta=90^{\circ}\right.$, i.e. equatorial alignment) with electric field polarizations both parallel and perpendicular to the axis of the particles $(\tau=0$ and $90^{\circ}$, respectively). For all of these shapes, absorption is much stronger than scattering and dominates the extinction; as with previous studies [5], we find that the spectra for $\theta=90^{\circ}, \tau=0^{\circ}$ aligned incident light show a resonance that, for the sphere, occurs at around $530 \mathrm{~nm}$. As the particle shape changes from sphere to rounded cylinder, this resonance remains largely unchanged in both position and magnitude. As the particle gains an indentation (at around E), this resonance moves to longer wavelengths, becoming initially stronger and then progressively weaker and then eventually disappearing. A heuristic interpretation of this behaviour is that the indentation perturbs the surface field, and the resonance at $530 \mathrm{~nm}$ moves towards longer wavelengths where the effect of indentation can be averaged out, until the indentation becomes dominant and the feature disappears from the range of wavelengths investigated. In the case of polarization perpendicular to the axis of the particles $\left(\tau=90^{\circ}\right)$, there is a broad resonance near $510 \mathrm{~nm}$ : as the particle moves away from spherical, this resonance becomes weaker while its wavelength very weakly blue-shifts. For both polarizations, the strongest absorption features of all particles are due to the onset of an absorption feature at short wavelengths.

The cross sections for $\theta=0^{\circ}$ incident light (along the axis of symmetry) are extremely similar to those for $\theta=90^{\circ}$ light with $\tau=90^{\circ}$ (see figure 5), with changes in the relative magnitude of the absorption cross sections being less than $\approx 4 \%$ (this maximum change occurring shortest wavelength).

The analysis of principal modes of the particles allows us to confirm the simple heuristic description of the $\theta=90^{\circ}$ incident light, and also provides a straightforward explanation for the close similarity between cross sections for $\theta=0^{\circ}$ light and $\theta=90^{\circ}$ incident light polarized $\tau=90^{\circ}$. We show in figures 6-9 the internal and scattering mode landscapes for selected particles, i.e. $\sin ^{-1}(\xi)$, the largest value of equations (2) and (3) for $\left|f^{0}\right|=1$ as a function of the order of the mode's principal cosines and wavelength of incident light. The shading of the traces overlaid on top of the landscapes shows, from the left to right column, the amplitudes excited 

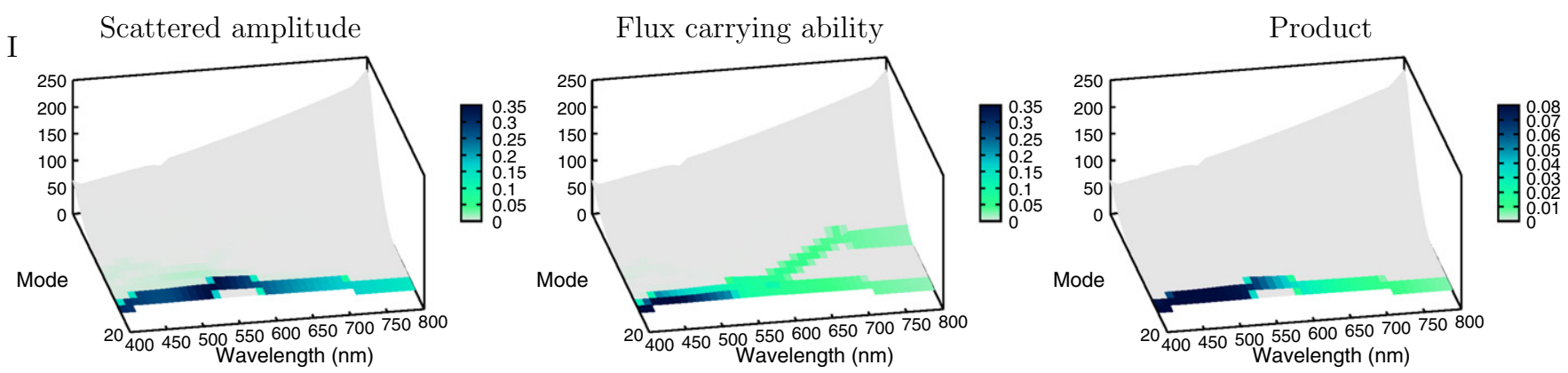

$\mathrm{E}$
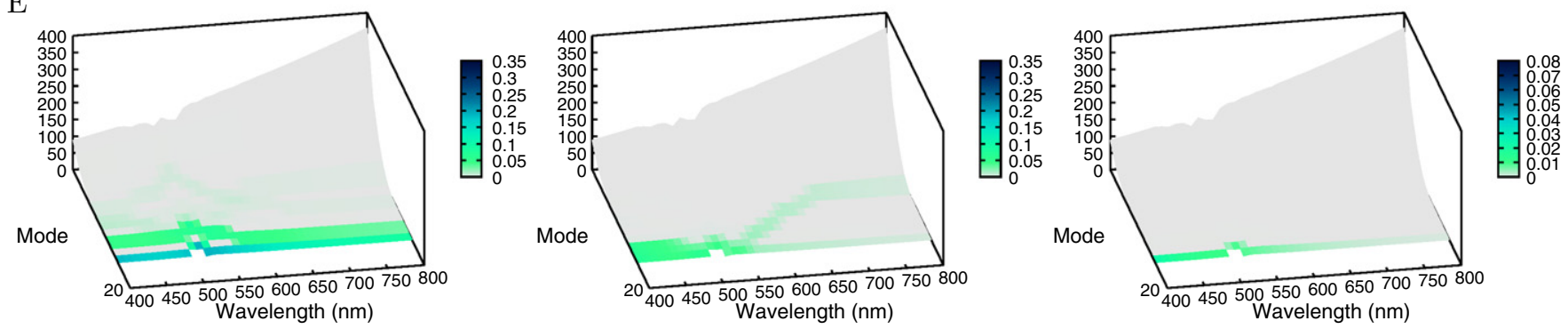

A
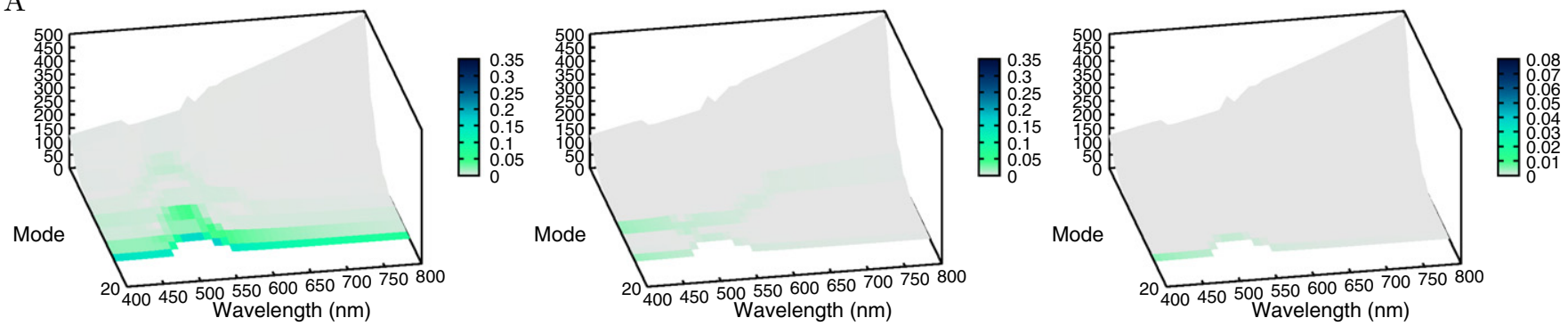

Figure 9. The $l_{z}= \pm 1$ scattering mode landscapes of the particles. These are again overlaid with traces showing the amplitudes of the modes excited by light with $\theta=0^{\circ}$ incidence and electric field perpendicular to the particle axis, their intrinsic ability to transport energy into the particle and the resulting effective flux being scattered away by each mode.

by the incident field (either $a_{n}^{i}$ or $a_{n}^{s}$, respectively), the modes' ability to transport energy (which is independent of the incident field) and the effective flux carried by the modes for the incident field considered (the product of the amplitude and flux carrying ability). In the case of light polarized along the axis of the particle $(\tau=0)$, interactions are dominated by the $l_{z}=0$ modes (figures 6 and 7), while for light polarized perpendicular to this axis (figures 8 and 9), only the $l_{z}= \pm 1$ modes show strong couplings; hence, only these channels are shown for these cases.

The feature at $530 \mathrm{~nm}$ for $\theta=0^{\circ}$ light polarized along the particle is due to a principal pair of internal and scattered modes, which are both strongly excited and can also transport energy (left and centre columns of figures 6 and 7) leading to the observed net transport of energy (rightmost column) responsible for figure 4(a). Moving from the spheroid (I) to the rounded cylinder $(\mathrm{E})$, the responsible mode pair becomes more aligned (climbing further up the landscape at its point of resonance) and leading to the enhancement of the absorption seen in figure $4(a)$ on moving from particle $\mathrm{J}$ to D. For particles A and B which have the deepest indentation, the mode paths are strongly deformed, with the formerly intense mode pair moving to low alignment, explaining the disappearance of this resonance in figure $4(a)$, by which point this mode pair is at resonance at $\sim 650 \mathrm{~nm}$. We can also see that the absorption onset at short wavelengths is clearly due to the group of several modes with both substantial excitation and flux-carrying abilities at short wavelengths. These mask the much weaker absorption feature present at $\sim 510 \mathrm{~nm}$ in all of the particles.

The scattering part of the principal mode pair corresponding to the absorbing feature at $530 \mathrm{~nm}$ is also able to transport some energy, but is too weak to be visible on the scale of figure 4(a) (an additional, even weaker active scattering mode at $\sim 510 \mathrm{~nm}$ is also resonant, but only for particle A). We can also see from figure 7 that there are several scattering principal modes of the particles that, while strongly coupled to the incident field, have a very poor ability to transport energy and so do not contribute significantly to the movement of energy away from the particle. These modes are particularly noticeable for the most indented cases (A and B) and are essential in fulfilling the electromagnetic boundary conditions, contributing substantially to the overall near-surface fields. Such modes are relevant to near-field applications of particles such as surface-enhanced spectroscopies, but can be easily missed when one observes only in the far field.

Turning now to incident fields with perpendicular polarization to the particle axis $\left(\tau=90^{\circ}\right)$, the feature at 
$510 \mathrm{~nm}$ is seen to be due to the shallow resonance of a weakly aligned pair (figure 8 ), with $l= \pm 1$ symmetry, while the short wavelength onset is seen to be due to the same mode gaining in flux-carrying ability as the wavelength decreases. There are very few other modes that show any excitation, and these are, as with the $\tau=0^{\circ}$ polarization, unable to transport energy.

Unlike the $l_{z}=0$ modes responsible for the features of the equatorial longitudinal polarized spectra $(\theta=90, \tau=0)$, the $l_{z}= \pm 1$ modes can be excited by $\theta=0^{\circ}$ incident light. On rotating the angle of incidence from $\theta=0^{\circ}$ to $\theta=90^{\circ}$ (while $\tau$ is kept constant at $90^{\circ}$ ) the excitation pattern of these modes is unchanged. This is due to the insensitivity with respect to orientation of the coupling between the $l_{z}= \pm 1$ modes and plane wave light with this polarization. For this geometry, the electric field component of the light remains constant as $\theta$ changes, while the change in the magnetic field is not important. The tiny residual differences shown in figure 5 are due to the small contributions from $l_{z}= \pm 2$ principal modes, which as with the $l_{z}=0$ modes are unable to couple to axially incident light and do show variation of coupling with incident angle.

\section{Conclusions}

We analyse the optical response of a set of gold nanoparticles with shapes ranging from a sphere to a composite particle made up from the fusion of two smaller ellipsoidal particles. The presence of indentations in the surface strongly modifies the structure of the modes up to the point that absorption and extinction cross section resonances disappear. This disappearance is accompanied by the excitation of several surface modes that have large surface fields, but little energy transport. These modes are relevant to many near-field and surface applications, even though they are very difficult to observe in the far field. The advantage of our approach is that we can disentangle the near and far-field features on the basis of the intrinsic modes of particles, allowing optimization of the input field with respect to given modes, and controlled enhancement of the far or near-field response.

\section{References}

[1] Wang D-S and Kerker M 1981 Enhanced Raman scattering by molecules adsorbed at the surface of colloidal spheroids Phys. Rev. B 24 1777-90
[2] Graham D and Goodacre R 2008 Chemical and bioanalytical applications of surface enhanced Raman scattering spectroscopy Chem. Soc. Rev. 37 883-4

[3] Aoki T, Dayan B, Wilcut E, Bowen W P, Parkins A S, Kippenberg T J, Vahala K J and Kimble H J 2006 Observation of strong coupling between one atom and a monolithic microresonator Nature 443 671-4

[4] Papoff F and Hourahine B 2011 Geometrical mie theory for resonances in nanoparticles of any shape Opt. Express $1921432-44$

[5] Romero I, Aizpurua J, Bryant G W and de Abajo F J G 2006 Plasmons in nearly touching metallic nanoparticles: singular response in the limit of touching dimers $O p t$. Express 14 9988-99

[6] McMahon J M, Henry A-I, Wustholz K L, Natan M J, Freeman R G, Van Duyne R P and Schatz G C 2009 Gold nanoparticle dimer plasmonics: finite element method calculations of the electromagnetic enhancement to surface-enhanced Raman spectroscopy Anal. Bioanal. Chem. 394 1819-25

[7] Holms K, Hourahine B and Papoff F 2009 Calculation of internal and scattered fields of axisymmetric nanoparticles at any point in space J. Opt. A: Pure Appl. Opt. 11054009

[8] Hannan E J 1961/1962 The general theory of canonical correlation and its relation to functional analysis $J$. Aust. Math. Soc. 2 229-42

[9] New G H C 1995 The origin of excess noise J. Mod. Opt. 42 799-810

[10] Firth W J and Yao A M 2005 Giant excess noise and transient gain in misaligned laser cavities Phys. Rev. Lett. 95073903

[11] Papoff F, D’Alessandro G and Oppo G-L 2008 State dependent pseudoresonances and excess noise Phys. Rev. Lett. 100123905

[12] D'Alessandro G and Laforet C B 2009 Giant noise amplification in synchronously pumped optical parametric oscillators Opt. Lett. 34 614-6

[13] D'Alessandro G and Papoff F 2009 Giant subthreshold amplification is synchronously pumped optical parametric oscillators Phys. Rev. A 40023804

[14] Gielis J 2003 A generic geometric transformation that unifies a wide range of natural and abstract shapes Am. J. Bot. $90333-8$

[15] Doicu A, Wriedt T and Eremin Y 2006 Light Scattering by Systems of Particles (Berlin: Springer)

[16] Etchegoin P G, Le Ru E C and Meyer M 2006 An analytic model for the optical properties of gold J. Chem. Phys. 125164705

[17] Etchegoin P G, Le Ru E C and Meyer M 2007 An analytic model for the optical properties of gold J. Chem. Phys. 127189901 (erratum) 